\title{
A Study of Interrelationship between Green Supplier Selection Enablers using ISM Methodology
}

\author{
Ratna Banerjee \\ School of Business, UPES \\ Dehradun
}

\author{
Remica Aggarwal \\ MIT-SOER, MIT - ADT University, \\ Pune, India
}

\author{
S. P. Singh \\ Department of Management \\ Studies, IIT Delhi \\ Delhi, India
}

\begin{abstract}
Appropriate supplier selection is an important and critical decision in supply chain management. It involves considering multiple conflicting criteria such as cost, delivery, lead- time etc. and hence researchers, for analyzing these criteria while selecting suppliers have adopted various multi-criteria methods or methodologies. In recent years, growing environmental awareness and environmental sustainability encourages consumers as well as organizations and enterprises to look for greener alternatives. The prime reason being that the supply chain activities and many logistics activities are the leading sources of carbon dioxide $\left(\mathrm{CO}_{2}\right)$ emission and environmental pollutants. The emergence of green supply chain paradigm lead to the incorporation of green or environment friendly criteria in supplier selection as well. Consideration of applying a mix of criteria i.e. traditional as well as green criteria while selecting an appropriate supplier is a challenge for any manufacturing enterprise. Present research paper focuses on this concept. The objectives in this paper are two folds: firstly it identify the necessary green supplier selection criteria based on author's own observation and extensive literature review and thereafter it studies the interrelationships and their dependence and driving relationships using an Interpretive Structural Modeling (ISM) technique. Analysis shows that criteria such as reputation, geographical location, financial position and quality planning are drivers as they have high driving power whereas green criteria such as technical expertise, green supplier image, environmental design, waste management system, recyclable material have high driving as well as high dependence power hence they are linkage criteria.
\end{abstract}

\section{Keywords}

Green Supply Chain, Supplier Selection Criteria, Interpretive Structural Modeling

\section{INTRODUCTION}

Change is the only constant now days. When we look towards consumer, we usually find that their needs, demands, tastes and preferences are changing. Consequently, coming up to the consumer's expectations, is a challenge for any manufacturing firm to maintain its credibility and good will in the market. Any successful organizations quickly recognizes the changing needs of its customers and adapt itself accordingly. Now days, consumers are becoming more health conscious and environment sensitive. Growing environmental awareness has successfully lead to the emergence of enterprises adopting greener alternatives. They are becoming smart in their choice of products. Also, the competition is increasing at an alarming rate. To stay ahead of the competitors, manufacturers are making smart choices to select the most appropriate supplier so that they can get their raw material or semi-finished products at a faster and at an affordable rate.
Not only that, various environment sensitive issues are also considered in almost every management aspect. Supply chain management is no exception. Hence while making judicious choice of their suppliers, various environmental criteria are also taken into consideration. The supply chains in such cases becomes green supply chains or sustainable supply chains which considers the three dimensions of sustainable development i.e. economic, environmental and social. Competitiveness is maintained through meeting customer needs and related economic criteria. Flow of goods, material, information as well as capital keeping in mind environmental risks and hazards and simultaneously seeking cooperation among companies i.e. socializing amongst themselves. Green supply chains therefore starts from the procurement of raw material and continue until disposal of the product in a greener manner. Present research focuses on identifying various green supplier selection criteria and then identifying their interrelationships and dependencies with the traditional supplier selection criteria using ISM methodology. The knowledge of interrelationships amongst various criteria helps the manufacturer to decide the appropriate supplier as per his needs and requirements simultaneously considering the competitive advantage over its competitors.

The paper is structured as follows: Section 2 presents the literature review on the green supplier selection criteria and the various methodologies used therein. Section 3 explain the ISM methodology and its applications in various fields of supply chain management. Section 4 empirically illustrate the problem and highlights various criteria taken into consideration for the case problem. Section 5 presents the ISM model for the case problem and the resulting diagraph. Managerial implications has been illustrated in section 6 and finally section 7 concludes the paper with future directions.

\section{LITERATURE REVIEW}

The emergence of the green supply chain paradigm and consequently supplier selection needs a rethinking of the traditional supplier selection criteria and to incorporate various green criteria and to study the inter-relationships amongst them so as to improve the environmental and sustainability performance of the whole supply system. Therefore, this section particularly devotes to an assessment of penetration of green issues in the literature regarding supplier selection problem.

In this paper, presently available literature has been analyzed using keywords such as 'supplier selection', 'green supplier selection', 'vendor selection' 'green supplier evaluation', 'environmental criteria for supplier selection' , 'green supply chain management', 'GSCM', 'carbon emissions' etc. have been searched over internet to get relevant abstracts of research papers or full research papers based on the topic. In order to broaden the timeline of the survey, the same and/or similar keywords has been utilized for performing a further 
search through the web-based GoogleScholar ${ }^{\mathrm{TM}}$ tool, Mendeley software and Research Gate engine. Thus, the resulting list of reference papers attempts to covers the last twenty years period.

\subsection{Traditional $v s$ green supplier selection criteria}

\subsubsection{Traditional supplier selection criteria}

Literatures such as $[1,2,3]$ etc. are reviewed to find the supplier selection criteria. Other than this, various websites such as google.com, Inderscience.com, Elsevier.com etc. were searched with keywords such as assessment criteria, supplier selection criteria, vendor selection etc. Quality , delivery, price and service flexibility being part of performance criteria; technical cooperation, financial status, company's image and reputation, geographical location and IT knowledge as part of business criteria and quality planning, quality assurance and quality control as part of quality system assessment.

\subsubsection{Green criteria for supplier selection}

Authors such as $[2,3,4]$ categorize green criteria into quantitative criteria (cost, waste or by-product and energy consumption) and qualitative criteria (which includes company image and reputation). Both $[5,6]$ has taken combined criteria (traditional as well as green criteria) in their researches to select appropriate suppliers.

The following table illustrates the prominent green supplier selection criteria and the corresponding authors with the publication year.

\begin{tabular}{|c|c|c|}
\hline $\begin{array}{l}\text { S. } \\
\text { No. }\end{array}$ & $\begin{array}{l}\text { Author and } \\
\text { Publication }\end{array}$ & Green criteria and the explanation \\
\hline 1. & {$[4,6,7]$} & Environmental management system \\
\hline 2. & [8], [9] & $\begin{array}{l}\text { Staff environmental training; training } \\
\text { related to carbon management }\end{array}$ \\
\hline 3. & [8] & $\begin{array}{c}\text { Use of environment friendly material; } \\
\text { environment friendly technology; } \\
\text { recyclable material / biodegradable } \\
\text { material }\end{array}$ \\
\hline 4. & $\begin{array}{c}{[2,3,4,} \\
7,8,13]\end{array}$ & Green supplier image \\
\hline 5. & {$[2,3,5,7,8]$} & $\begin{array}{l}\text { Social responsibility and environmental } \\
\text { competencies }\end{array}$ \\
\hline 6. & [13] & $\begin{array}{l}\text { Waste management system ; waste } \\
\text { recovery disposal ; waste water and solid } \\
\text { waste }\end{array}$ \\
\hline 7. & {$[2,3,7,8]$} & Environmental design ; eco design \\
\hline
\end{tabular}

\subsection{Methods or Methodologies for the green supplier selection}

Looking at the journal contributions to the research topic, following table gives the list of number of papers against the prominently searched journals. The table illustrates the various methods or methodologies used by the researchers for selecting and ranking various green supplier selection criteria.

\begin{tabular}{|c|c|c|}
\hline S. No. & $\begin{array}{l}\text { List of } \\
\text { authors } \\
\text { contributing } \\
\text { to the topic }\end{array}$ & $\begin{array}{l}\text { Concept of the paper \& the } \\
\text { methodologies used }\end{array}$ \\
\hline 1. & {$[15]$} & $\begin{array}{l}\text { Qualitative methodology based } \\
\text { on the Ishikawa diagram }\end{array}$ \\
\hline 2. & [14] & $\begin{array}{l}\text { Methodologies based on } \\
\text { AHP/ANP by[12] }\end{array}$ \\
\hline 3. & [15] & $\begin{array}{l}\text { Methodologies based on } \\
\text { AHP/ANP by [12] }\end{array}$ \\
\hline 4. & [7] & $\begin{array}{l}\text { Methodologies based on } \\
\text { AHP/ANP by [12] }\end{array}$ \\
\hline 5. & {$[5,6,9]$} & $\begin{array}{l}\text { Methodologies based on } \\
\text { AHP/ANP by [12] }\end{array}$ \\
\hline 6. & [5] & Croquet Integral approach \\
\hline 7. & [17] & Rough set theory \\
\hline 8. & [3] & Fuzzy set theory \\
\hline 9. & [1] & $\begin{array}{l}\text { Fuzzy association rule mining } \\
\text { approach }\end{array}$ \\
\hline 10. & [4] & Hybrid methodology of AHP \\
\hline 11. & {$[8]$} & $\begin{array}{l}\text { Environmental performance of } \\
\text { suppliers using Fuzzy multi- } \\
\text { criteria approach }\end{array}$ \\
\hline 12. & {$[10,11]$} & $\begin{array}{l}\text { fuzzy multi-criteria decision } \\
\text { framework under incomplete } \\
\text { information }\end{array}$ \\
\hline 13. & {$[10,11]$} & $\begin{array}{l}\text { Fuzzy DEMATEL, fuzzy ANP \& } \\
\text { fuzzy TOPSIS for green supplier } \\
\text { selection }\end{array}$ \\
\hline 14. & [6] & $\begin{array}{l}\text { Carbon management model using } \\
\text { DEMATEL approach }\end{array}$ \\
\hline 15. & [15] & $\begin{array}{l}\text { Fuzzy axiomatic design approach } \\
\text { for supplier selection }\end{array}$ \\
\hline 16. & [15] & $\begin{array}{l}\text { Integrated fuzzy multi-criteria } \\
\text { and multi-objective programming } \\
\text { approach }\end{array}$ \\
\hline 17. & [19] & $\begin{array}{l}\text { Fuzzy AHP \& fuzzy multi- } \\
\text { objective programming }\end{array}$ \\
\hline 18. & {$[20]$} & $\begin{array}{l}\text { ANP model for collaborative } \\
\mathrm{CO}_{2} \text { reduction management }\end{array}$ \\
\hline 19. & [21] & Pareto genetic algorithms \\
\hline
\end{tabular}

\section{INTERPRETIVE STRUCTURAL MODELLING METHODOLOGY}

Warfield [22] proposed the technique Interpretive Structural Modeling or ISM that enables the researcher to map the interrelationships amongst various criteria involved in a complex situation. A structured hierarchy graph is thus obtained from the set of unique interrelated variables. Group or expert judgement decides whether and how the items are related. 
ISM works with the following steps:

1. Identification of elements, which are relevant to the decision maker's problems and issues.

2. Establishing the contextual relationship between elements and with respect to which pairs of elements will be examine.

3. Developing a structural self-interaction matrix (SSIM): Four symbols viz. $V, A, X \& O$ are used for establishing the relationships. Here $\mathrm{V}$ stands for the relation from $i$ to $j$; A for the relation from $j$ to $i$; X for both direction relations from $i$ to $j$ and $j$ to $i$; and $\mathrm{O}$ if there is no valid relationships between the variables.

4. Formation of Reachability Matrix: Using SSIM, initial reachability matrix can be formed which has all values in binary form. Rule of transitivity is usually checked and any transitivity is removed while creating final reachability matrix.

5. Creating reachability and antecedent set: From the final reachability matrix, the reachability set and antecedent set for each criterion and for each element is found (Warfield (1974)). The element for which the reachability and intersection sets are the same is the toplevel element.

6. Level Partition Matrix: The whole process of partitioning is based on establishing the precedence relationships and arranging the elements in a topological order.

7. Classification of variables: Variables are classified based on relative driving power and dependence power in to the categories like autonomous, dependent, driver and linkage.

7. Development of Diagraph/ISM.

\subsection{Applications of ISM in supply chain management}

Kannan \& Haq [7] analyze the interaction of criteria and subcriteria that are used to select the supplier for the built-inorder supply chain environment in the original equipment manufacturing company using ISM approach. A case of southern India is taken to illustrate the methodology. Luthra S. et al. [23] identified various barriers to implement green supply chain management in an Indian automobile industry using the technique of interpretive structural modeling. Dubey [24] build a green supply chain management theory using interpretive structural modeling (ISM) and identified the enablers of green supply chain as well as barriers to green supply chain using exhaustive literature review and expert opinion. Dube \& Gwaande [25] identified the various barriers to green supply chain and studied their interrelationships through ISM methodology.

\section{DEVELOPMENT OF ISM MODEL}

In this section, ISM model is developed for the green supplier selection process. Out of the total variables, 18 important criteria are considered that is a mix of traditional supplier selection criteria as well as green criteria. Amongst the traditional supplier selection criteria are price/ pricing charged by supplier $(\mathrm{P})$; service flexibility ( $\mathrm{SFl}$ ); on time delivery (De); quality of products and services offered by supplier (Qu); supplier's technical expertise / capability (TE); financial position of supplier (FP); supplier's reputation (Re) ; geographical location of supplier (Lo) ; quality planning at supplier's end (QP); quality assurance (QA) and quality control (QC). Amongst the green supplier selection criteria are environmental management system (EMS) ; environmental training to staff (ETS); use of environment friendly/recyclable material (RM); green supplier image (GSI) ; social responsibility \& environmental competencies (SR/EC); Waste management system / waste recovery disposal / waste water and solid waste (WMS) ; effective environmental design /eco design(ED).

\subsection{Construction of Structural Self- Interaction Matrix (SSIM)}

This matrix gives the pairwise relationship between two variables i.e. $i$ and $j$ based on VAXO. Around 20 challenges $v i z$. are now studied for possible inter-relationship amongst them using ISM methodology viz. Price $(\mathrm{P})$; technical expertise / capability (TE); service flexibility (SFl); delivery (De); quality $(\mathrm{Qu})$; financial position (FP); reputation $(\mathrm{Re})$; geographical location (Lo) ; quality planning (QP); quality assurance (QA) and quality control (QC). Amongst the green supplier selection criteria are environmental management system (EMS) ; staff environmental training (SET); use of environment friendly/recyclable material (RM); green supplier image (GSI) ; social responsibility \& environmental competencies (SR/EC); waste management system / waste recovery disposal / waste water and solid waste (WMS) ; environmental design /eco design(ED)

Explanation: Price $(\mathrm{P})$ of the service provided by supplier includes on time delivery (De), technical competence or expertise (TE), service flexibility (SF). Waste management system (WMS) ensures good quality products as well as optimal utilization of resources (concept of lean six sigma). Similarly, use of recyclable material will affect the price $(\mathrm{P})$ charged by the supplier or the cost of service. Technical expertise (TE) may lead to effective use of resources or raw material. There is a greater probability that a financially sound (FS) and reputed (Re) supplier will encourage or lead to effective environmental management system (EMS) , environmental training to staff (ETS) as it will not only lead to his /her good will as well as create green supplier image (GSI) in the society which is a plus for his business with clients. It is seen now days that big brands or reputed manufacturers and suppliers are maintaining their corporate social responsibility image (SR) and serves as an inspiration for SME's. Socially responsible (SR) criteria as well as green supplier image (GSI) will add to the reputation (Re) of supplier. Geographical location of supplier may affect the supplier decisions towards environmental or green criteria. Those suppliers who are placed in more congested metropolitan cities are because of strict government regulation or taxes are responding positively towards the methods to combat increasing carbon emissions levels. A strong and well planned environmental management system (EMS) can lead to effective environmental training to staff (ETS), green supplier image (GSI), effective use of biodegradable materials (RM) , effective waste management system (WMS)(and vice versa).

\subsection{Initial Reachability Matrix}

There are some transitive relationships and they are updated in the final reachability matrix. They are from $1 \rightarrow 7,1 \rightarrow 10$, $2 \rightarrow 7,2 \rightarrow 8,2 \rightarrow 9,2 \rightarrow 12,2 \rightarrow 13,2 \rightarrow 15,2 \rightarrow 16,2 \rightarrow 18$, $3 \rightarrow 7,3 \rightarrow 10,3 \rightarrow 11,4 \rightarrow 5,4 \rightarrow 7,4 \rightarrow 10,7 \rightarrow 5,7 \rightarrow 16,8 \rightarrow 2$, $8 \rightarrow 9, \quad 14 \rightarrow 2,14 \rightarrow 3, \quad 14 \rightarrow 5, \quad 14 \rightarrow 10, \quad 14 \rightarrow 11, \quad 14 \rightarrow 12$, $14 \rightarrow 13,15 \rightarrow 2 \ldots .15 \rightarrow 7,15 \rightarrow 15 \ldots 15 \rightarrow 18,16 \rightarrow 2 \ldots .16 \rightarrow 6$ $, 16 \rightarrow 10, \ldots 16 \rightarrow 14, \quad 17 \rightarrow 2 \ldots . .17 \rightarrow 18,18 \rightarrow 4, . .18 \rightarrow 6$, $18 \rightarrow 10 \ldots .18 \rightarrow 18$. These transitivity relationships have been 
incorporated to obtain the final reachability matrix as follows. All these values are changed to 1 in the final reachability matrix. (highlighted in yellow). Based on driving power and dependencies, these factors may be classified in to four groups of autonomous, dependent, linkage and independent (driver) factors.

\begin{tabular}{|c|c|c|c|c|c|c|c|c|c|c|c|c|c|c|c|c|c|c|c|}
\hline & & 1 & 2 & 3 & 4 & 5 & 6 & 7 & 8 & 9 & 10 & 11 & 12 & 13 & 14 & 15 & 16 & 17 & 18 \\
\hline & & $\mathrm{P}$ & TE & SFl & $\mathrm{De}$ & $\mathrm{Qu}$ & FP & $\mathrm{Re}$ & GLo & QP & $\overline{\mathrm{QA}}$ & $\mathrm{QC}$ & EMS & ETS & RM & GSI & $\begin{array}{l}\text { SR } \\
\end{array}$ & WMS & ED \\
\hline 1 & $\mathrm{P}$ & & $\mathrm{V}$ & $\mathrm{V}$ & $\mathrm{V}$ & $\mathrm{V}$ & A & A & A & $\mathrm{O}$ & $\mathrm{O}$ & $\mathrm{O}$ & $\mathrm{O}$ & $\mathrm{O}$ & $\mathrm{A}$ & A & A & $\mathrm{O}$ & $\mathrm{O}$ \\
\hline 2 & TE & & & $\mathrm{V}$ & V & $\mathrm{V}$ & $\mathrm{V}$ & $\mathrm{A}$ & $\mathrm{O}$ & $\mathrm{A}$ & $\mathrm{V}$ & $\mathrm{V}$ & $\mathrm{O}$ & $\mathrm{O}$ & $\mathrm{V}$ & $\mathrm{O}$ & $\mathrm{O}$ & $\mathrm{V}$ & $\mathrm{O}$ \\
\hline 3 & SFl & & & & V & $\mathrm{V}$ & $\mathrm{A}$ & A & $\mathrm{A}$ & $\mathrm{O}$ & $\mathrm{O}$ & $\mathrm{O}$ & $\mathrm{O}$ & $\mathrm{O}$ & $\mathrm{O}$ & $\mathrm{O}$ & $\mathrm{O}$ & $\mathrm{O}$ & $\mathrm{O}$ \\
\hline 4 & $\mathrm{De}$ & & & & & $\mathrm{V}$ & $\bar{A}$ & $\bar{A}$ & A & $\bar{A}$ & $\mathrm{X}$ & $\mathrm{O}$ & $\mathrm{O}$ & $\bar{O}$ & $\bar{A}$ & $\mathrm{O}$ & $\mathrm{O}$ & $\mathrm{O}$ & $\mathrm{O}$ \\
\hline 5 & $\mathrm{Qu}$ & & & & & & $\mathrm{A}$ & $\mathrm{V}$ & $\mathrm{A}$ & $\mathrm{A}$ & V & $\mathrm{A}$ & $\mathrm{A}$ & $\mathrm{A}$ & $\mathrm{A}$ & $\mathrm{A}$ & $\mathrm{A}$ & $\mathrm{A}$ & A \\
\hline 6 & FP & & & & & & & $\mathrm{X}$ & $\mathrm{O}$ & $\mathrm{V}$ & $\mathrm{V}$ & $\mathrm{V}$ & $\mathrm{V}$ & $\mathrm{V}$ & $\mathrm{V}$ & $\mathrm{V}$ & $\mathrm{V}$ & $\mathrm{V}$ & $\mathrm{V}$ \\
\hline 7 & $\operatorname{Re}$ & & & & & & & & $\mathrm{O}$ & V & V & V & V & V & V & A & A & $\mathrm{V}$ & $\mathrm{V}$ \\
\hline 8 & GLo & & & & & & & & & $\mathrm{O}$ & $\mathrm{O}$ & $\mathrm{O}$ & V & V & V & V & V & V & $\mathrm{V}$ \\
\hline 9 & QP & & & & & & & & & & $\mathrm{V}$ & V & $\mathrm{V}$ & $\mathrm{X}$ & $\mathrm{X}$ & $\mathrm{X}$ & $\mathrm{X}$ & $\mathrm{X}$ & $\mathrm{X}$ \\
\hline 10 & $\overline{\mathrm{QA}}$ & & & & & & & & & & & A & $\mathrm{O}$ & $\mathrm{O}$ & $\mathrm{O}$ & $\mathrm{O}$ & $\mathrm{O}$ & $\mathrm{O}$ & $\mathrm{O}$ \\
\hline 11 & $\mathrm{QC}$ & & & & & & & & & & & & $\mathrm{O}$ & $\mathrm{O}$ & $\mathrm{O}$ & $\mathrm{O}$ & $\mathrm{O}$ & $\mathrm{O}$ & $\mathrm{O}$ \\
\hline 12 & EMS & & & & & & & & & & & & & $\bar{V}$ & $\mathrm{~V}$ & $\mathrm{~V}$ & $\mathrm{~V}$ & $\bar{X}$ & $\bar{V}$ \\
\hline 13 & ETS & & & & & & & & & & & & & & $\mathrm{V}$ & $\mathrm{V}$ & $\mathrm{V}$ & $\mathrm{V}$ & $\mathrm{O}$ \\
\hline 14 & RM & & & & & & & & & & & & & & & $\mathrm{V}$ & $\mathrm{V}$ & $\mathrm{V}$ & $\mathrm{O}$ \\
\hline 15 & GSI & & & & & & & & & & & & & & & & $\mathrm{V}$ & $\mathrm{V}$ & $\mathrm{O}$ \\
\hline 16 & SR & & & & & & & & & & & & & & & & & $\mathrm{O}$ & $\mathrm{O}$ \\
\hline 17 & WMS & & & & & & & & & & & & & & & & & & $\mathrm{V}$ \\
\hline 18 & ED & & & & & & & & & & & & & & & & & & \\
\hline
\end{tabular}

Fig 1: SSIM matrix for pair wise relationship amongst supplier selection criteria

\begin{tabular}{|c|c|c|c|c|c|c|c|c|c|c|c|c|c|c|c|c|c|c|c|}
\hline & & 1 & 2 & 3 & 4 & 5 & 6 & 7 & 8 & 9 & 10 & 11 & 12 & 13 & 14 & 15 & 16 & 17 & 18 \\
\hline & & P & TE & SFl & De & Qu & FP & Re & GLo & QP & QA & QC & EMS & ETS & RM & GSI & SR & WMS & ED \\
\hline 1 & P & 1 & 0 & 1 & 1 & 1 & 0 & 0 & 0 & 0 & 0 & 0 & 0 & 0 & 0 & 0 & 0 & 0 & 0 \\
\hline 2 & TE & 1 & 1 & 1 & 1 & 1 & 1 & 0 & 0 & 0 & 1 & 1 & 0 & 0 & 1 & 0 & 0 & 1 & 0 \\
\hline 3 & SFl & 0 & 0 & 1 & 1 & 1 & 0 & 0 & 0 & 0 & 0 & 0 & 0 & 0 & 0 & 0 & 0 & 0 & 0 \\
\hline 4 & De & 0 & 0 & 0 & 1 & 1 & 0 & 0 & 0 & 0 & 1 & 0 & 0 & 0 & 0 & 0 & 0 & 0 & 0 \\
\hline 5 & Qu & 0 & 0 & 0 & 0 & 1 & 0 & 1 & 0 & 0 & 1 & 0 & 0 & 0 & 0 & 0 & 0 & 0 & 0 \\
\hline 6 & FP & 1 & 0 & 1 & 1 & 1 & 1 & 1 & 1 & 1 & 1 & 1 & 1 & 1 & 1 & 1 & 1 & 1 & 1 \\
\hline 7 & Re & 1 & 1 & 1 & 1 & 0 & 1 & 1 & 0 & 1 & 1 & 1 & 1 & 1 & 1 & 0 & 0 & 1 & 1 \\
\hline 8 & GLo & 1 & 0 & 1 & 1 & 1 & 0 & 0 & 1 & 0 & 0 & 0 & 1 & 1 & 1 & 1 & 1 & 1 & 1 \\
\hline 9 & QP & 0 & 1 & 0 & 1 & 1 & 0 & 0 & 0 & 1 & 1 & 1 & 1 & 1 & 1 & 1 & 1 & 1 & 1 \\
\hline 10 & QA & 0 & 0 & 0 & 1 & 0 & 0 & 0 & 0 & 0 & 1 & 0 & 0 & 0 & 0 & 0 & 0 & 0 & 0 \\
\hline 11 & QC & 0 & 0 & 0 & 0 & 0 & 0 & 0 & 0 & 0 & 1 & 1 & 0 & 0 & 0 & 0 & 0 & 0 & 0 \\
\hline 12 & EMS & 0 & 0 & 0 & 0 & 0 & 0 & 0 & 0 & 0 & 0 & 0 & 1 & 1 & 1 & 1 & 1 & 1 & 1 \\
\hline 13 & ETS & 0 & 0 & 0 & 0 & 0 & 0 & 0 & 0 & 1 & 0 & 0 & 0 & 1 & 1 & 1 & 1 & 1 & 0 \\
\hline 14 & RM & 1 & 0 & 0 & 1 & 0 & 0 & 0 & 0 & 1 & 0 & 0 & 0 & 0 & 1 & 1 & 1 & 1 & 0 \\
\hline 15 & GSI & 1 & 0 & 0 & 0 & 0 & 0 & 1 & 0 & 1 & 0 & 0 & 0 & 0 & 0 & 1 & 1 & 1 & 0 \\
\hline 16 & SR & 1 & 0 & 0 & 0 & 0 & 0 & 1 & 0 & 1 & 0 & 0 & 0 & 0 & 0 & 0 & 1 & 0 & 0 \\
\hline 17 & WMS & 0 & 0 & 0 & 0 & 0 & 0 & 0 & 0 & 1 & 0 & 0 & 1 & 0 & 0 & 0 & 0 & 1 & 1 \\
\hline 18 & ED & 0 & 0 & 0 & 0 & 0 & 0 & 0 & 0 & 1 & 0 & 0 & 0 & 0 & 0 & 0 & 0 & 0 & 1 \\
\hline
\end{tabular}

Fig 2: Initial reachability matrix 


\begin{tabular}{|c|c|c|c|c|c|c|c|c|c|c|c|c|c|c|c|c|c|c|c|c|}
\hline & & 1 & 2 & 3 & 4 & 5 & 6 & 7 & 8 & 9 & 10 & 11 & 12 & 13 & 14 & 15 & 16 & 17 & 18 & $\begin{array}{l}\text { D. } \\
\text { P }\end{array}$ \\
\hline & & $P$ & $\begin{array}{l}\mathrm{T} \\
\mathrm{E}\end{array}$ & $\begin{array}{c}\text { SF } \\
1\end{array}$ & $\begin{array}{l}\mathrm{D} \\
\mathrm{e}\end{array}$ & $\begin{array}{l}\mathrm{Q} \\
\mathrm{u}\end{array}$ & $\begin{array}{l}F \\
P\end{array}$ & $\begin{array}{c}\mathrm{R} \\
\mathrm{e}\end{array}$ & $\begin{array}{c}\text { GL } \\
0\end{array}$ & $\begin{array}{l}\mathrm{Q} \\
\mathrm{P}\end{array}$ & $\begin{array}{l}\mathrm{Q} \\
\mathrm{A}\end{array}$ & $\begin{array}{l}\mathrm{Q} \\
\mathrm{C}\end{array}$ & $\begin{array}{c}\text { EM } \\
\text { S }\end{array}$ & $\begin{array}{c}\text { ET } \\
\text { S }\end{array}$ & $\begin{array}{l}\mathrm{R} \\
\mathrm{M}\end{array}$ & $\begin{array}{c}\text { GS } \\
\text { I }\end{array}$ & $\begin{array}{l}\mathrm{S} \\
\mathrm{R}\end{array}$ & $\begin{array}{c}\text { WM } \\
\text { S }\end{array}$ & $\begin{array}{l}E \\
D\end{array}$ & \\
\hline 1 & $\mathrm{P}$ & 1 & 0 & 1 & 1 & 1 & 0 & 1 & 0 & 0 & 1 & 0 & 0 & 0 & 0 & 0 & 0 & 0 & 0 & 6 \\
\hline 2 & $\mathrm{TE}$ & 1 & 1 & 1 & 1 & 1 & 1 & 1 & 1 & 1 & 1 & 1 & 1 & 1 & 1 & 1 & 1 & 1 & 1 & 18 \\
\hline 3 & SFl & 0 & 0 & 1 & 1 & 1 & 0 & 1 & 0 & 0 & 1 & 1 & 0 & 0 & 1 & 0 & 0 & 0 & 0 & 7 \\
\hline 4 & De & 0 & 0 & 0 & 1 & 1 & 0 & 1 & 0 & 0 & 1 & 0 & 0 & 0 & 0 & 0 & 0 & 0 & 0 & 4 \\
\hline 5 & $\mathrm{Qu}$ & 0 & 0 & 0 & 1 & 1 & 0 & 1 & 0 & 0 & 1 & 0 & 0 & 0 & 0 & 0 & 0 & 0 & 0 & 4 \\
\hline 6 & FP & 1 & 1 & 1 & 1 & 1 & 1 & 1 & 1 & 1 & 1 & 1 & 1 & 1 & 1 & 1 & 1 & 1 & 1 & 18 \\
\hline 7 & $\operatorname{Re}$ & 1 & 1 & 1 & 1 & 1 & 1 & 1 & 0 & 1 & 1 & 1 & 1 & 1 & 1 & 0 & 1 & 1 & 1 & 16 \\
\hline 8 & GLo & 1 & 1 & 1 & 1 & 1 & 0 & 0 & 1 & 1 & 0 & 0 & 1 & 1 & 1 & 1 & 1 & 1 & 1 & 15 \\
\hline 9 & QP & 0 & 1 & 0 & 1 & 1 & 0 & 0 & 0 & 1 & 1 & 1 & 1 & 1 & 1 & 1 & 1 & 1 & 1 & 13 \\
\hline $\begin{array}{l}1 \\
0\end{array}$ & QA & 0 & 0 & 0 & 1 & 1 & 0 & 0 & 0 & 0 & 1 & 0 & 0 & 0 & 0 & 0 & 0 & 0 & 0 & 3 \\
\hline $\begin{array}{l}1 \\
1\end{array}$ & $\overline{Q C}$ & 0 & 0 & 0 & 1 & 1 & 0 & 0 & 0 & 0 & 1 & 1 & 0 & 0 & 0 & 0 & 0 & 0 & 0 & 4 \\
\hline 1 & EMS & 0 & 0 & 0 & 0 & 0 & 0 & 0 & 0 & 0 & 0 & 0 & 1 & 1 & 1 & 1 & 1 & 1 & 1 & 7 \\
\hline 1 & ETS & 0 & 0 & 0 & 0 & 0 & 0 & 0 & 0 & 1 & 0 & 0 & 0 & 1 & 1 & 1 & 1 & 1 & 0 & 6 \\
\hline 1 & RM & 1 & 1 & 1 & 1 & 1 & 0 & 0 & 0 & 1 & 1 & 1 & 1 & 1 & 1 & 1 & 1 & 1 & 0 & 14 \\
\hline 1 & GSI & 1 & 1 & 1 & 1 & 1 & 1 & 1 & 0 & 1 & 1 & 1 & 1 & 1 & 1 & 1 & 1 & 1 & 1 & 17 \\
\hline 1 & SR & 1 & 1 & 1 & 1 & 1 & 1 & 1 & 0 & 1 & 1 & 1 & 1 & 1 & 1 & 0 & 1 & 1 & 1 & 16 \\
\hline 1 & WM & 0 & 1 & 0 & 1 & 1 & 1 & 0 & 0 & 0 & 1 & 1 & 1 & 1 & 1 & 1 & 1 & 1 & 1 & 13 \\
\hline I & & & & & & & & & & & & & & & & & & & & \\
\hline \multirow{2}{*}{$\begin{array}{l}1 \\
8\end{array}$} & ED & 0 & 1 & 0 & 1 & 1 & 1 & 0 & 0 & 0 & 1 & 1 & 1 & 1 & 1 & 1 & 1 & 1 & 1 & 13 \\
\hline & De. P & 8 & 10 & 9 & 16 & 16 & 7 & 9 & 3 & 9 & 15 & 11 & 11 & 12 & 13 & 10 & 12 & 12 & 10 & \\
\hline
\end{tabular}

\section{Fig 3 : Final reachability matrix}

D.P : Driving power ; De.P : dependence power

\subsection{Level Partition}

From the final reachability matrix, reachability and final antecedent set for each factor are found. The elements for which the reachability and intersection sets are same are the top-level element in the ISM hierarchy. After the identification of top level element, it is separated out from the other elements and the process continues for next level of elements. Reachability set, antecedent set, intersection set along with different level for elements have been shown below in table 4 .

Table 4.3.1: Iteration I

\begin{tabular}{|l|c|c|c|c|}
\hline S. No. & $\begin{array}{c}\text { Reachabili } \\
\text { ty set }\end{array}$ & Antecedent set & $\begin{array}{c}\text { Intersec } \\
\text { tion set }\end{array}$ & Level \\
\hline $\mathbf{1 .}$ & $\mathbf{5}$ & $\begin{array}{c}1,2,3,4,5,6,7,8,9, \\
10,11,13,14,15,16,\end{array}$ & $\mathbf{5}$ & \\
. & & & \\
\hline
\end{tabular}

\begin{tabular}{|c|c|c|c|c|}
\hline & & 17,18 & & \\
\hline 2. & 4,10 & $\begin{array}{c}1,2,3,4,5,6,7,9 \\
10,11,13,14,15,16 \\
17,18\end{array}$ & 4,10 & \\
\hline 3. & $13,14,15$ & $\begin{array}{c}2,6,8,9,12,13,14 \\
15,17,18\end{array}$ & $13,14,15$ & \\
\hline 4. & $13,16,17$ & $\begin{array}{c}2,6,8,9,12,13,14, \\
15,16,17,18\end{array}$ & $13,16,17$ & \\
\hline 5. & 12,13 & $\begin{array}{c}2,6,7,8,9,12,14,15 \\
16,17,18\end{array}$ & 12 & $\mathbf{I}$ \\
\hline 6. & 10,11 & $\begin{array}{c}2,3,6,7,9,11,14,15 \\
16,17,18\end{array}$ & 11 & \\
\hline
\end{tabular}




\begin{tabular}{|c|c|c|c|}
\hline 7. & 3,7 & $1,2,3,6,7,15,16$ & 3,7 \\
\hline 8. & 16,18 & $\begin{array}{c}2,6,8,9,12,15,16 \\
17,18\end{array}$ & 16,18 \\
\hline 9. & 7,10 & $\begin{array}{c}1,2,3,4,5,6,7,15 \\
16\end{array}$ & 7 \\
\hline $\begin{array}{r}10 . \\
1 .\end{array}$ & $4,5,9$ & $2,6,7,8,9,14,15,16$ & 9 \\
\hline $\begin{aligned} 11 . \\
2 .\end{aligned}$ & 5,7 & $\begin{array}{c}1,2,3,4,5,6,7,15 \\
16\end{array}$ & 5,7 \\
\hline $\begin{array}{r}12 . \\
3 .\end{array}$ & 3,4 & $\begin{array}{c}1,2,3,6,7,8,14,15,1 \\
6\end{array}$ & 3 \\
\hline $\begin{array}{r}13 . \\
4 .\end{array}$ & 2,4 & $\begin{array}{c}2,6,7,8,9,14,15,16, \\
17,18\end{array}$ & 2 \\
\hline $\begin{array}{r}14 . \\
5 .\end{array}$ & 1,4 & $1,2,6,7,8,14,15,16$ & 1 \\
\hline $\begin{array}{r}15 . \\
6 .\end{array}$ & 6,9 & $2,6,7,15,16$ & 6 \\
\hline $\begin{array}{r}16 . \\
7 .\end{array}$ & 1,9 & $1,6,7,8,14,15,16$ & 1 \\
\hline $\begin{array}{r}17 . \\
8 .\end{array}$ & 8,12 & $1,6,8$ & 8 \\
\hline $\begin{array}{r}18 . \\
9 .\end{array}$ & $1,2,3$ & $2,7,14,15,16$ & 2 \\
\hline $\begin{array}{r}19 . \\
0 .\end{array}$ & $1,6,7,18$ & $2,6,7,15,16$ & 6,7 \\
\hline $\begin{array}{r}20 . \\
1 .\end{array}$ & 6,10 & $2,6,7,15,16,17,18$ & 6 \\
\hline
\end{tabular}

Table 4.3.2: Iteration II

\begin{tabular}{|c|c|c|c|c|}
\hline S. No. & $\begin{array}{c}\text { Reach } \\
\text { ability } \\
\text { set }\end{array}$ & Antecedent set & $\begin{array}{l}\text { Intersec } \\
\text { tion set }\end{array}$ & Level \\
\hline 2. & 4,10 & $\begin{array}{c}1,2,3,4,6,7,9,10,11,13,1 \\
4,15,16,17,18\end{array}$ & 4,10 & \multirow{10}{*}{ II } \\
\hline 3. & $\begin{array}{l}13,14 \\
15\end{array}$ & $\begin{array}{c}2,6,8,9,12,13,14,15,17 \\
18\end{array}$ & $13,14,15$ & \\
\hline 4. & $\begin{array}{c}13,16 \\
17\end{array}$ & $\begin{array}{c}2,6,8,9,12,13,14,15,16 \\
17,18\end{array}$ & $13,16,17$ & \\
\hline 5. & 12,13 & $\begin{array}{c}2,6,7,8,9,12,14,15,16,1 \\
7,18\end{array}$ & 12 & \\
\hline 6. & 10,11 & $\begin{array}{c}2,3,6,7,9,11,14,15,16,1 \\
7,18\end{array}$ & 11 & \\
\hline 7. & 3,7 & $1,2,3,6,7,15,16$ & 3,7 & \\
\hline 8. & 16,18 & $2,6,8,9,12,15,16,17,18$ & 16,18 & \\
\hline 9. & 7,10 & $1,2,3,4,6,7,15,16$ & 7 & \\
\hline 10. & 4,9 & $2,6,7,8,9,14,15,16$ & 9 & \\
\hline 11 & 7 & $1,2,3,4,6,7,15,16$ & 7 & \\
\hline
\end{tabular}

\begin{tabular}{|c|c|c|c|}
\hline 12 & 3,4 & $\begin{array}{c}1,2,3,6,7,8,14,15,16 \\
3\end{array}$ \\
\hline 13 & 2,4 & $\begin{array}{c}2,6,7,8,9,14,15,16,17,1 \\
8\end{array}$ & 2 \\
\hline 14. & 1,4 & $1,2,6,7,8,14,15,16$ & 1 \\
\hline 15. & 6,9 & $2,6,7,15,16$ & 6 \\
\hline 16. & 1,9 & $1,6,7,8,14,15,16$ & 1 \\
\hline 17. & 8,12 & $1,6,8$ & 8 \\
\hline 18. & $1,2,3$ & $2,7,14,15,16$ & 2 \\
\hline 19. & $\begin{array}{c}1,6,7,1 \\
8\end{array}$ & $2,6,7,15,16$ & 6,7 \\
\hline 20. & 6,10 & $2,6,7,15,16,17,18$ & 6 \\
\hline
\end{tabular}

Table 4.3.3: Iteration III

\begin{tabular}{|c|c|c|c|c|}
\hline S. No. & $\begin{array}{l}\text { Reachabili } \\
\text { ty set }\end{array}$ & Antecedent set & $\begin{array}{l}\text { Intersec } \\
\text { tion set }\end{array}$ & Level \\
\hline 3. & $13,14,15$ & $\begin{array}{c}2,6,8,9,12,13,14,15 \\
, 17,18\end{array}$ & $13,14,15$ & \multirow{18}{*}{ III } \\
\hline 4. & $13,16,17$ & $\begin{array}{c}2,6,8,9,12,13,14,15 \\
, 16,17,18\end{array}$ & $13,16,17$ & \\
\hline 5. & 12,13 & $\begin{array}{c}2,6,7,8,9,12,14,15 \\
16,17,18\end{array}$ & 12 & \\
\hline 6. & 11 & $\begin{array}{c}2,3,6,7,9,11,14,15 \\
16,17,18\end{array}$ & 11 & \\
\hline 7. & 3,7 & $1,2,3,6,7,15,16$ & 3,7 & \\
\hline 8. & 16,18 & $\begin{array}{c}2,6,7,8,9,12,15,16 \\
17,18\end{array}$ & 16,18 & \\
\hline 9. & 7 & $1,2,3,4,5,6,7,15,16$ & 7 & \\
\hline 10. & 9 & $2,6,7,8,9,14,15,16$ & 9 & \\
\hline 11 & 7 & $1,2,3,5,6,7,15,16$ & 7 & \\
\hline 12 & 3 & $\begin{array}{c}1,2,3,6,7,8,14,15,1 \\
6\end{array}$ & 3 & \\
\hline 13 & 2 & $\begin{array}{c}2,6,7,8,9,14,15,16 \\
17,18\end{array}$ & 2 & \\
\hline 14. & 1 & $1,2,6,7,8,14,15,16$ & 1 & \\
\hline 15. & 6,9 & $2,6,7,15,16$ & 6 & \\
\hline 16. & 1,9 & $1,6,7,8,14,15,16$ & 1 & \\
\hline 17. & 8,12 & $1,6,8$ & 8 & \\
\hline 18. & $1,2,3$ & $2,7,14,15,16$ & 2 & \\
\hline 19. & $1,6,7,18$ & $2,6,7,15,16$ & 6,7 & \\
\hline 20. & 6 & $2,6,7,15,16,17,18$ & 6 & \\
\hline
\end{tabular}

Table 4.3.4: Iteration IV

\begin{tabular}{|c|c|c|c|c|}
\hline $\begin{array}{c}\text { S. } \\
\text { No. }\end{array}$ & $\begin{array}{c}\text { Reachab } \\
\text { ility set }\end{array}$ & Antecedent set & $\begin{array}{c}\text { Intersec } \\
\text { tion set }\end{array}$ & Level \\
\hline 3. & 14,15 & $2,6,8,9,12,14,15,18$ & 14,15 & \\
\cline { 1 - 4 } 5. & $\mathbf{1 2}$ & $2,6,7,8,9,12,14,15,18$ & $\mathbf{1 2}$ & \\
\hline
\end{tabular}




\begin{tabular}{|c|c|c|c|c|}
\hline 6. & 11 & $2,3,6,7,9,11,14,15,18$ & 11 & \multirow{15}{*}{ IV } \\
\hline 7. & 3,7 & $1,2,3,6,7,15$ & 3,7 & \\
\hline 8. & 18 & $2,6,7,8,9,12,15,18$ & 16,18 & \\
\hline 9. & 7 & $1,2,3,4,5,6,7,15$ & 7 & \\
\hline 10. & $4,5,9$ & $2,6,7,8,9,14,15$ & 9 & \\
\hline 11. & 5,7 & $1,2,3,4,5,6,7,15$ & 5,7 & \\
\hline 12. & 3 & $1,2,3,6,7,8,14,15$ & 3 & \\
\hline 13. & 2 & $2,6,7,8,9,14,15,18$ & 2 & \\
\hline 14. & 1 & $1,2,6,7,8,14,15$ & 1 & \\
\hline 15. & 6,9 & $2,6,7,15$ & 6 & \\
\hline 16. & 1,9 & $1,6,7,14,15$ & 1 & \\
\hline 17. & 8,12 & $1,6,8$ & 8 & \\
\hline 18. & $1,2,3$ & $2,7,14,15$ & 2 & \\
\hline 19. & $1,6,7,18$ & $2,6,7,15$ & 6,7 & \\
\hline 20. & 6 & $2,6,7,15,18$ & 6 & \\
\hline
\end{tabular}

Table 4.3.5: Iteration V

\begin{tabular}{|c|c|c|c|c|}
\hline $\begin{array}{l}\text { S. } \\
\text { No. }\end{array}$ & $\begin{array}{c}\text { Reachab } \\
\text { ility set }\end{array}$ & Antecedent set & $\begin{array}{c}\text { Intersec } \\
\text { tion set }\end{array}$ & Level \\
\hline 3. & 14,15 & $2,6,8,9,14,15,17,18$ & 14,15 & \multirow{15}{*}{ V } \\
\hline 7. & 3,7 & $1,2,3,6,7,15$ & 3,7 & \\
\hline 8. & 18 & $2,6,7,8,9,15,18$ & 18 & \\
\hline 9. & 7 & $1,2,3,4,5,6,7,15$ & 7 & \\
\hline 10. & 9 & $2,6,7,8,9,14,15$ & 9 & \\
\hline 11. & 7 & $1,2,3,4,5,6,7,15$ & 7 & \\
\hline 12. & 3 & $1,2,3,6,7,8,14,15$ & 3 & \\
\hline 13. & 2 & $2,6,7,8,9,14,15,18$ & 2 & \\
\hline 14. & 1 & $1,2,6,7,8,14,15$ & 1 & \\
\hline 15. & 6,9 & $2,6,7,15,16$ & 6 & \\
\hline 16. & 1,9 & $1,6,7,14,15,16$ & 1 & \\
\hline 17. & 8,12 & $1,6,8$ & 8 & \\
\hline 18. & $1,2,3$ & $2,7,14,15,16$ & 2 & \\
\hline 19. & $1,6,7,18$ & $2,6,7,15$ & 6,7 & \\
\hline 20. & 6 & $2,6,7,15,18$ & 6 & \\
\hline
\end{tabular}

\begin{tabular}{|c|c|c|c|c|}
\hline 11. & 7 & $1,3,6,7$ & 7 & \multirow{9}{*}{ VI } \\
\hline 12. & 3 & $1,3,6,7,8$ & 3 & \\
\hline 14. & 1 & $1,6,7,8$ & 1 & \\
\hline 15. & 6,9 & 6,7 & 6 & \\
\hline 16. & 1,9 & $1,6,7$ & 1 & \\
\hline 17. & 8,12 & $1,6,8$ & 8 & \\
\hline 18. & 1,3 & 7 & 2 & \\
\hline 19. & $1,6,7,18$ & 6,7 & 6,7 & \\
\hline 20. & 6 & $6,7,18$ & 6 & \\
\hline
\end{tabular}

Table 4.3.7: Iteration VII

\begin{tabular}{|c|c|c|c|c|}
\hline $\begin{array}{l}\text { Sr. } \\
\text { No. }\end{array}$ & $\begin{array}{c}\text { Reachab } \\
\text { ility set }\end{array}$ & Antecedent set & $\begin{array}{c}\text { Intersec } \\
\text { tion set }\end{array}$ & Level \\
\hline 7. & 7 & $1,6,7$ & 7 & \multirow{11}{*}{ VII } \\
\hline 9. & 7 & $1,6,7$ & 7 & \\
\hline 10. & 9 & $6,7,8,9$ & 9 & \\
\hline 11. & 7 & $1,6,7$ & 7 & \\
\hline 14. & 1 & $1,6,7,8$ & 1 & \\
\hline 15. & 6,9 & 6,7 & 6 & \\
\hline 16. & 1,9 & $1,6,7$ & 1 & \\
\hline 17. & 8,12 & $1,6,8$ & 8 & \\
\hline 18. & 1 & 7 & 2 & \\
\hline 19. & $1,6,7,18$ & 6,7 & 6,7 & \\
\hline 20. & 6 & $6,7,18$ & 6 & \\
\hline
\end{tabular}

Table 4.3.8: Iteration VIII

\begin{tabular}{|c|c|c|c|c|}
\hline $\begin{array}{l}\text { Sr. } \\
\text { No. }\end{array}$ & $\begin{array}{c}\text { Reachab } \\
\text { ility set }\end{array}$ & Antecedent set & $\begin{array}{c}\text { Intersec } \\
\text { tion set }\end{array}$ & Level \\
\hline 7. & 7 & 6,7 & 7 & \multirow{7}{*}{ VIII } \\
\hline 9. & 7 & 6,7 & 7 & \\
\hline 11. & 7 & 6,7 & 7 & \\
\hline 15. & 6 & 6,7 & 6 & \\
\hline 17. & 8 & 6,8 & 8 & \\
\hline 19. & 6,7 & 6,7 & 6,7 & \\
\hline 20. & 6 & 6,7 & 6 & \\
\hline
\end{tabular}

Table 4.3.6: Iteration VI

\begin{tabular}{|c|c|c|c|c|}
\hline $\begin{array}{c}\text { S. } \\
\text { No. }\end{array}$ & $\begin{array}{c}\text { Reachab } \\
\text { ility set }\end{array}$ & Antecedent set & $\begin{array}{c}\text { Intersec } \\
\text { tion set }\end{array}$ & Level \\
\hline 7. & 3,7 & $1,3,6,7$ & 3,7 & \\
\cline { 1 - 4 } 8. & $\mathbf{1 8}$ & $6,7,8,9,18$ & $\mathbf{1 8}$ & \\
\cline { 1 - 4 } 9. & 7 & $1,3,6,7$ & 7 & \\
\cline { 1 - 4 } 10. & 9 & $6,7,8,9$ & 9 & \\
\hline
\end{tabular}

\subsection{Classification of factors}

In this section, the critical success factors described earlier are classified in to four clusters viz. autonomous factor, dependent factors, linkage factors and independent factors .This has been mentioned in Table 5.5 below. As it can be seen that the criteria such as SFI and $\mathrm{P}$ falls under autonomous category. They have medium dependence and driving power .Criteria such as QC, ETS, EMS, De, Qu, QA falls under high dependence and low to medium driving 
power. They are dependent criteria. Similarly, Re, GLo, FP , QP have low dependence but high driving power and hence they are drivers whereas TE, SR, RM , GSI , ED and WMS falls for high driving as well as dependence power. Hence, they are linkage criteria.

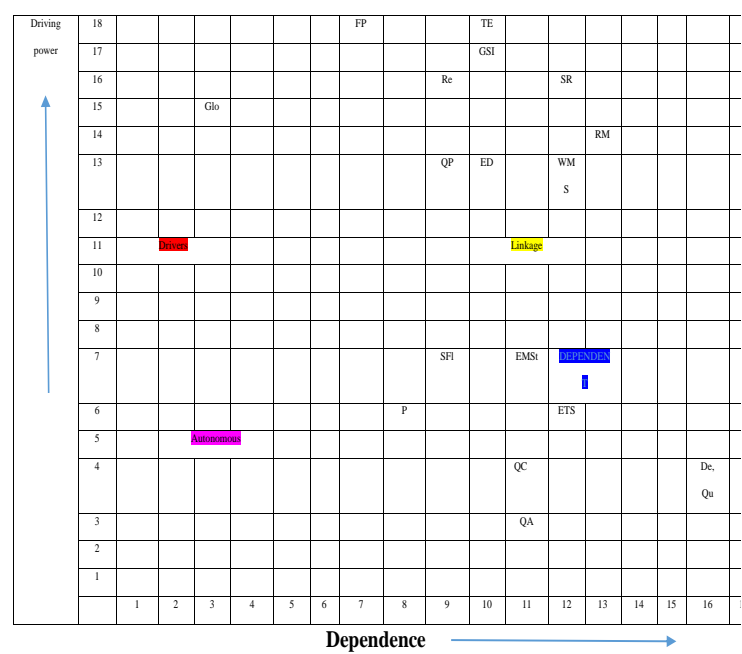

Fig. 4.Driving Power and Dependence Diagram

\subsection{ISM Diagraph}

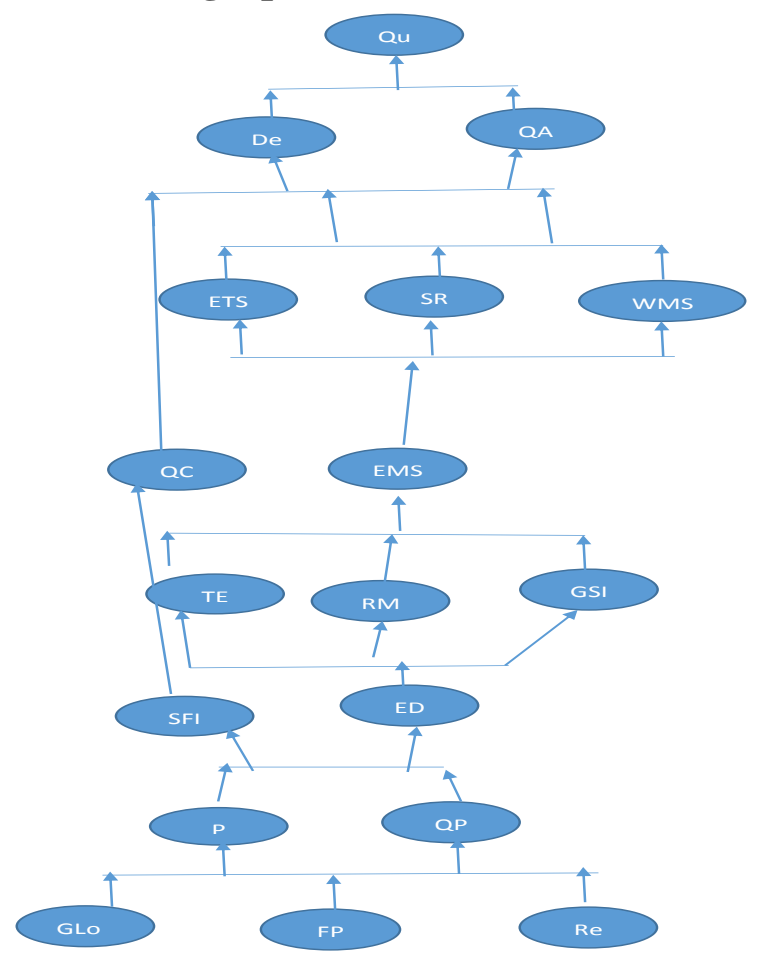

Fig 5 : ISM Diagraph

\section{RESEARCH IMPLICATIONS}

The study findings and discussion of the study would be beneficial for the academicians, research scholars, practitioners, managers and policy makers. The study of interrelationship and dependency relationship between various traditional supply chain criteria as well as green criteria would benefit academicians and research scholars, as this study gives further insights in this field and there are less numbers of researches available especially in Indian context. Managers and practitioners can understand the opportunities, which are emerging in the field of green supply chain and there is need of much more focus on it. These interrelationships are quite insightful for managers also as they can accordingly plan and select appropriate suppliers keeping in mind both economic as well as environmental criteria.

\section{CONCLUSIONS AND DIRECTIONS FOR FURTHER RESEARCH}

Present research work focuses on suggesting an ISM methodology for studying the interrelationship between supply chain as well as green criteria. Incorporation of green criteria is quite new and emerging from Indian perspective as consumers are getting environment conscious. The research topic can further be extended to include hybrid methodologies such as fuzzy DEMATEL or TOPSIS

\section{ACKNOWLEDGMENTS}

Our thanks to the anonymous reviewers whose comments have helped us in improving the manuscript .

\section{REFERENCES}

[1] Jain, V., Wadhwa, S. and Deshmukh, S. G. 2007. Supplier selection using fuzzy association rules mining approach. International Journal of Production Research, $45(6), 1323-1353$

[2] Humphreys, P.K., Wong, Y.K. Chan, F.T.S. 2003 Integrating environmental criteria into the supplier selection process, Journal of Materials Processing Technology, 138 (1-3), 349-56.

[3] Humphreys, P.K., McCloskey, A., McIvor, R. Maguire, L., Glackin, C. 2006. Employing dynamic fuzzy membership functions to assess environmental performance in the supplier selection process. International Journal of Production Research, 44, 2379 2419.

[4] Lee A.H.I., Kang, H.K. Hsu, C.F., Hung, H.C. 2009. A green supplier selection model for high-tech industry. Expert Systems with Applications, 36, 7917-7927.

[5] Feyzioğlu, O., Büyüközkan, G. 2008. Evaluation of Suppliers Environmental Management Performances by a Fuzzy Compromise Ranking Technique", Journal of Multiple-Valued Logic and Soft Computing, 14(3-5), 309-323.

[6] Hsu, C., Hu A. 2009. Applying hazardous substance management to supplier selection using analytic network process .Journal of Cleaner Production, 255-264.

[7] Kannan, G., Haq, A. N., Sasikumar P., Arunachalam, S., 2008.Analysis and selection of green suppliers using interpretative structural modelling and analytic hierarchy process. International Journal of Management and Decision Making, 9(2), $163-182$.

[8] Awasthi A., Chauhan , S.S. and Goyal , S.K. 2010. A fuzzy multi-criteria approach for evaluating environmental performance of suppliers" , International Journal of Production Economics, 126 (2), 370-378.

[9] Hsu, C.W, Kuo, T.C., Chen, S.H. and Hu, A.H. 2013 Using DEMATEL to develop a carbon management model of supplier selection in green supply chain management. Journal of cleaner production 56:164-172.

[10] Buyukozkan G. and Cifci, G. 2011. A novel fuzzy multicriteria decision framework for sustainable supplier 
selection with incomplete information. Journal of computers in industry, 62 (2), 164-174.

[11] Buyukozkan, G. and Cifci G. 2012 . A novel hybrid MCDM approach based on fuzzy DEMATEL, fuzzy ANP and fuzzy TOPSIS to evaluate green suppliers. Expert System and applications, 39:3000-3011.

[12] Saaty, T.L. 1980. The Analytic Hierarchy Process. McGraw-Hill, New York

[13] Handfield, R., Steven, R., Srouft, R., \& Melnyk, S. A. 2002 . Applying environmental criteria to supplier assessment: A study in the application of the analytical hierarchy process. European Journal of Operational Research, 141, 70-87.

[14] Enarsson, L. 1998. Evaluation of suppliers: how to consider the environment", International Journal of Physical Distribution and Logistics Management, 28(1), 5-17.

[15] Kannan , D. Roohollah, K.L.O , Jafarian A. and Diabat, A. 2013. Integrated fuzzy multi criteria decision making method and multi-objective programming approach for supplier selection and order allocation in a green supply chain. Journal of cleaner production, 47, 355-367.

[16] Lu, L. Y.Y., Wu, C.H. and Kuo, T.-C. 2007. Environmental principles applicable to green supplier evaluation by using multi-objective decision analysis. International Journal of Production Research, 45(18), $4317-4331$

[17] Bai, C., Sarkis, J., 2010 . Green supplier development: Analytical evaluation using rough set theory, Journal of Cleaner Production, in press.

[18] Kuo R.J., Wang, Y.C. and Tien, F.C. 2010. Integration of artificial neural network and MADA methods for green supplier selection, Journal of Cleaner Production, 18(12), 1161-117.

[19] K Shaw, K., Shankar, R. , Yadav, S.S. and Thakur, L.S. 2012. Supplier selection using fuzzy AHP and fuzzy multi-objective linear programming for developing low carbon supply chain . Expert System \& applications, 39, 8182-8192.

[20] Theiben, S. and Spinler, S. 2014. Strategic analysis of manufacturer-supplier partnerships: An ANP model for collaborative $\mathrm{CO}_{2}$ reduction management. European journal of operational research, 233:383-397.

[21] Zhang, Y., Tao, F., Laili, Y., Hou , B. , Lv, L. and Zhang, L. 2013. Green partner selection in virtual enterprise based on pareto genetic algorithms", International Journal of Advanced Manufacturing technology , 67, 2109-2125.

[22] Warfield, J. N. 1974. Developing interconnection matrices in structural modeling. IEEE Transactions on System, Man, and Cybernetics, SMC-4 (1), 81-87.

[23] Luthra, S., Kumar, V. and Haleem, A. 2011. Barriers to implement green supply chain management in automobile industry using interpretive structural modeling technique: an Indian perspective. Journal of Industrial engineering \& Management, 4(2), 231-257.

[24] Dubey, R. , Gunasekaran , A. Fosso, S. and Wamba, S.B. 2015. Building Theory of Green Supply Chain Management using Total Interpretive Structural Modeling. IFAC-Papers Online, Elsevier Science Direct , 48 (3) , 1688-1694.

[25] Dube, A.S. and Gawande , R.S. 2016. Analysis of green supply chain barriers using integrated ISM-fuzzy MICMAC approach. Benchmarking: An International Journal, 23(6), $1558-1578$. 